\title{
Evaluación de los aprendizajes en la educación superior intercultural
}

\author{
Learning assessment in intercultural higher education
}

William Oswaldo Flores López ${ }^{1}$

\section{Resumen}

La evaluación de los aprendizajes en la educación superior intercultural es complementaria con los saberes y haceres de los pueblos, en el sentido, que la evaluación es fundamental para construir una comunidad de aprendizaje (saber ser); para aprender en comunidad (saber hacer); para formar en y para la comunidad (saber conocer); y para acompañar a la comunidad (saber convivir). Desde esta perspectiva, la evaluación de los aprendizajes es un proceso que describe los conocimientos, saberes y prácticas que las personas adquieren durante sus trayectos de vidas. Se concluye, que una evaluación de los aprendizajes inclusiva para poblaciones en contextos multiculturales, tiene que contribuir a la reducción de situaciones de desigualdad y exclusión social, así como, promover una evaluación para todos y con todos, teniendo de referencia el reconocimiento de la diversidad de conocimientos y experiencias, y la incorporación de tecnologías accesibles que posibiliten un aprendizaje para toda la vida.

Palabras clave: Evaluación; Aprendizajes; Educación Superior Intercultural; Saberes y Haceres; Inclusiva; Diversidad.

\section{Abstract}

Learning assessment in intercultural higher education is complementary to the knowledge and doings of the people, in the sense that evaluation is fundamental to build a learning community (know to be); to learn in community (know to do); to form in and for the community (know to know); and to accompany the community (know to coexist). From this perspective, the learning assessment is a process that describes the knowledge, wisdom and practices that people acquire during their lifetimes. It is concluded that an inclusive learning assessment for populations in multicultural contexts must contribute to the reduction of situations of inequality and social exclusion, as well as promoting an assessment for all and with all, taking as reference the recognition of diversity of knowledge and experiences, and the incorporation of accessible technologies that enable lifelong learning.

1 Doctor en Educación. Profesor Investigador de la Universidad de las Regiones Autónomas de la Costa Caribe Nicaragüense. Correo: william.flores@uraccan.edu.ni (1): https://orcid.org/oooo-ooo2-1016-1620

Recibido: 15/04/2018 Aprobado: 01/09/2018 
Key Words: Evaluation; Learnings; Intercultural Higher Education; Knowledge and Doings; Inclusive; Diversity.

\section{Introducción}

La evaluación de los aprendizajes en la educación superior intercultural es fundamental para avanzar en el reconocimiento de los conocimientos, saberes y prácticas de los pueblos. En este contexto, la evaluación de los aprendizajes debe considerar las características individuales y socioculturales de los pueblos, teniendo de referencia la diversidad de las poblaciones en el acto educativo. Sin embargo, en la educación superior de Nicaragua, existe una deserción del estudiantado universitario por factores como: emotivos, académicos, económicos, motivacionales, normativos, ambientes familiares, orientación profesional, absentismos por género (femenino), marginación cultural, étnico, lingüísticos, discapacidad y procesos de acompañamiento al estudiantado en su formación (Flores et al., 2016).

Además, existe una crisis de aprendizaje que perjudica gravemente a los jóvenes desfavorecidos y en riesgo de exclusión social, caracterizada por: docentes pocos calificados y desmotivados; estudiantes poco preparados; gestión de las escuelas que no tienen efecto en la enseñanza ni en el aprendizaje; insumos escolares que no tienen efecto en la enseñanza ni en el aprendizaje (Banco Mundial, 2018). Es por ello, que surge la interrogante, ¿De qué manera una evaluación de los aprendizajes es inclusiva para poblaciones en contextos multiculturales? Para profundizar en una respuesta a esta interrogante, se parte de la conceptualización de la evaluación de los aprendizajes y posteriormente se proponen pautas para una evaluación de los aprendizajes en contextos multiculturales con eje principal la interculturalidad en las poblaciones.

\section{Fundamentación Teórica}

La evaluación esta orientada a evaluar las competencias del estudiantado teniendo de referencia, problemas del contexto profesional, social disciplinar e investigativo, estableciendo evidencias e indicadores, buscando determinar el grado de desarrollo en sus tres dimensiones (efectivo-motivacional, cognoscitiva y actitudinal) para brindar retroalimentación en torno a fortalezas y aspectos a mejorar en los procesos de metacognición, procesual y actitudinal a partir de la reflexión problematizadora desde la investigación acción participativa (López-Mairena \& Trillo-Alonso 2013).

Flores y Salinas (2012) definen a la evaluación como un proceso de mejora, así como, para regular los aprendizajes y su carácter interactivo requiere establecer un buen sistema de comunicación entre el estudiantes y profesores. Flores y Rojas (2013) proponen que en la evaluación de los aprendizajes se tiene que incorporar tecnologías de la información y comunicación porque permiten desarrollar capacidades diferentes tales como: la reflexión, búsqueda de la información concreta, facilidad para interactuar 
entre profesor y estudiante, capacidad para analizar de manera crítica y constructiva, y sobre todo el autoaprendizaje y que al ser evaluados de esta manera el estudiantado se siente motivados.

En Gaitán-Hernández, Lacayo-Robles y Flores (2014) se afirma que la evaluación permite contrastar los resultados de las experiencias de aprendizaje con el desempeño personal, valorando su esfuerzo, cumplimiento y calidad de las asignaciones, el punto de partida de la evaluación de los aprendizajes es diagnóstica puntual y procedimental en relación con los objetivos e indicadores de aprendizaje. En Flores (2015) se propone que los instrumentos de evaluación deben responder a los objetivos de aprendizajes, así como, al perfil profesional del estudiantado, es decir, la práctica evaluativa tiene que contextualizar el quehacer profesional del estudiantado, en concreto una evaluación con prácticas de la vida cotidiana de los discentes.

La evaluación de los aprendizajes es el proceso por medio del cual se recolecta evidencia que permite establecer los logros de los estudiantes en cuanto a sus aprendizajes para poder emitir juicios de valor y tomar decisiones para mejorar tanto la enseñanza como el aprendizaje del estudiantado (Amador-Núñez, Reyes-Gómez, \& Flores, 2015). Además, la evaluación es un proceso didáctico, coherente y sistemático que articula lo cognitivo y afectivo para la construcción de aprendizajes en contextos del mundo real (Flores \& Auzmendi, 2015). En cambio, Flores y Olivar-Molina (2016) comunican que, en los procesos de evaluación de los aprendizajes, las estrategias de evaluación que el profesorado implementa podrían influir en las actitudes de sus futuros estudiantes, por tales razones, es necesario, promover espacios de formación para docentes que atiende poblaciones diversas, teniendo de referencia los componentes afectivos, cognitivos, valor y dificultad que se manifiestan en el acto educativo.

A partir de las ideas anteriores, se puede concluir que, la evaluación de los aprendizajes en la educación superior intercultural tienen que contribuir a la superación de las desigualdades de hombres y mujeres procedente de contextos multiculturales, es decir, reducir situaciones de desigualdad y exclusión social, así como, promover una evaluación para todos y con todos, teniendo de referencia el reconocimiento de la diversidad de saberes y prácticas, y la incorporación de tecnologías accesibles que posibiliten un aprendizaje para toda la vida.

\section{Innovaciones Educativas}

La evaluación es un proceso que describe los conocimientos, saberes y prácticas que las personas adquieren durante los trayectos de sus vidas. Es decir, el mecanismo que busca hacer las valoraciones sobre los niveles de apropiación en los procesos de aprendizaje. La evaluación de los aprendizajes en contextos multiculturales y plurilingües es todo un proceso integral que parte de los conocimientos y saberes previos de los estudiantes, de la implementación de un proceso de enseñanza pertinente al 
contexto como lo es el contexto multicultural y plurilingüe, del proceso de enseñanza mismo implementando metodologías acertadas, pertinente y en armonía con el contexto. La evaluación de los aprendizajes mide o evalúa el nivel de apropiación de los conocimientos adquiridos en dicho proceso.

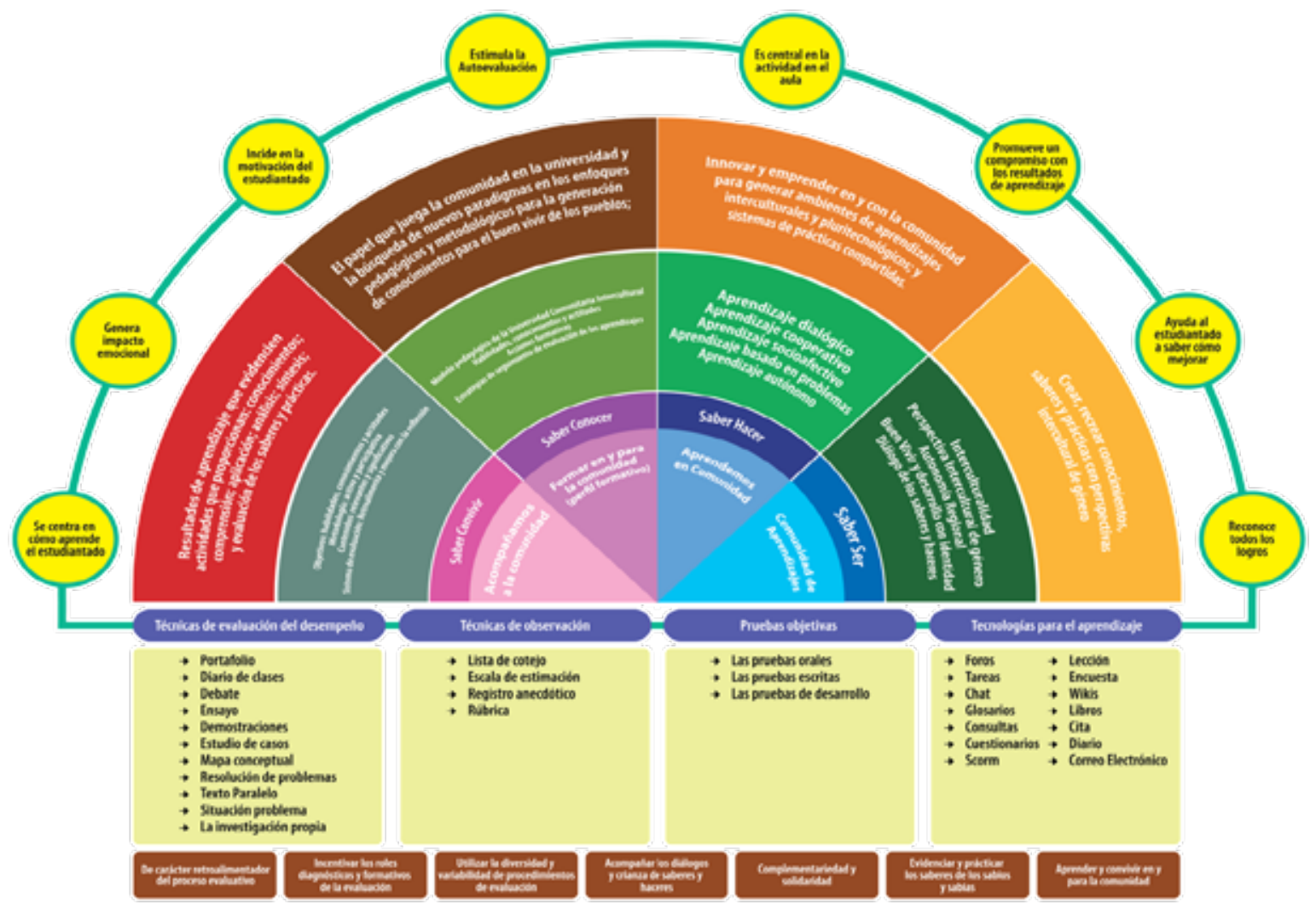

Figura 1: Sistema de evaluación de los aprendizajes (Flores, 2017).

Entonces, la evaluación de los aprendizajes en la educación superior intercultural, toma de referencia la diversidad de saberes de las poblaciones indígenas, afrodescendientes, mestizas y comunidades étnicas, según Tobón (2008), estos son:

- Saber ser: es la capacidad de la autodirección, responsabilidad, solución de problemas, toma de decisiones y valores propios del estudiantado para percibir a los demás y vivir en comunidad.

- Saber hacer: está relacionado con el uso exitoso de los conocimientos disciplinarios adquiridos y ahora aplicados al contexto donde se desenvuelve el estudiantado. 


\section{INNOVACIONES EDUCATIVAS}

- Saber conocer: se relaciona con los conocimientos que se adquieren en torno a una o varias disciplinas.

- Saber convivir: está relacionado con los valores asociados a la capacidad para establecer y desarrollar relaciones sociales en todos los espacios en donde le corresponda interactuar al estudiantado.

Es por ello, que la evaluación de los aprendizajes en la educación intercultural inicia con la interacción y reconocimiento de la diversidad de aprendizaje de los pueblos, que participan en el aula intercultural, siendo esta, un espacio de diálogo para la creación, recreación, diseminación e intercambio de conocimientos, saberes y prácticas a través de procesos de participación democráticos e inclusivos donde interactúan los conocimientos propios de los pueblos en una ambiente de aprendizaje con incorporación de tecnologías de aprendizaje.

\subsection{La evaluación de los aprendizajes desde una perspectiva multicultural}

Evaluar desde y para la diversidad se parte de la base de que cada persona implica singularidad, cada estudiante llega a la universidad con andamiaje y un equipamiento cultural, afectivo, social e intelectual particular, único. Está condicionado por las experiencias personales, el ambiente familiar y sociocultural en que vive y sus características individuales. La diversidad significa diferentes respuestas, pero también diversos caminos para llegar a ellas (Santos-Guerra, 1999; Roncal, 2005). En el contexto de poblaciones multiculturales, la evaluación ha de considerar las diferencias individuales y socioculturales y a partir del reconocimiento de la diversidad en sí misma.

Se hace necesario considerar, los aspectos siguientes para mejorar los procesos evaluativos: lo importante de la evaluación para poblaciones indígenas radica en el acercamiento del individuo a las normas y pautas culturales que permitan salvaguardar la identidad del pueblo; una de las características de la evaluación para poblaciones indígenas, afrodescendientes y mestizas es que se da en una relación estrecha de padrehijo, madre-hija y otros parientes en el momento mismo en el que hijo o hija realiza una determinada actividad. Se evalúa diariamente; la evaluación para poblaciones en contextos multiculturales también se caracteriza por su carácter cualitativo y es una evaluación integral porque considera todos los ámbitos de la vida del estudiantado: afectivo, cognoscitivo y psicomotor; el aspecto valorativo en la evaluación para poblaciones en contextos multiculturales es una responsabilidad colectiva, pues participan todos los miembros de la comunidad, tanto mayores como grupos de igual de edad; y las comunidades de práctica son las que valoran o evalúan la práctica especifica que lleva a cabo una persona.

En este sentido, la evaluación de los aprendizajes se presenta como una posibilidad de desplazamiento de perspectiva en contra de las prácticas más habituales en evaluación. Sus ideas centrales se resumen en principios como: 


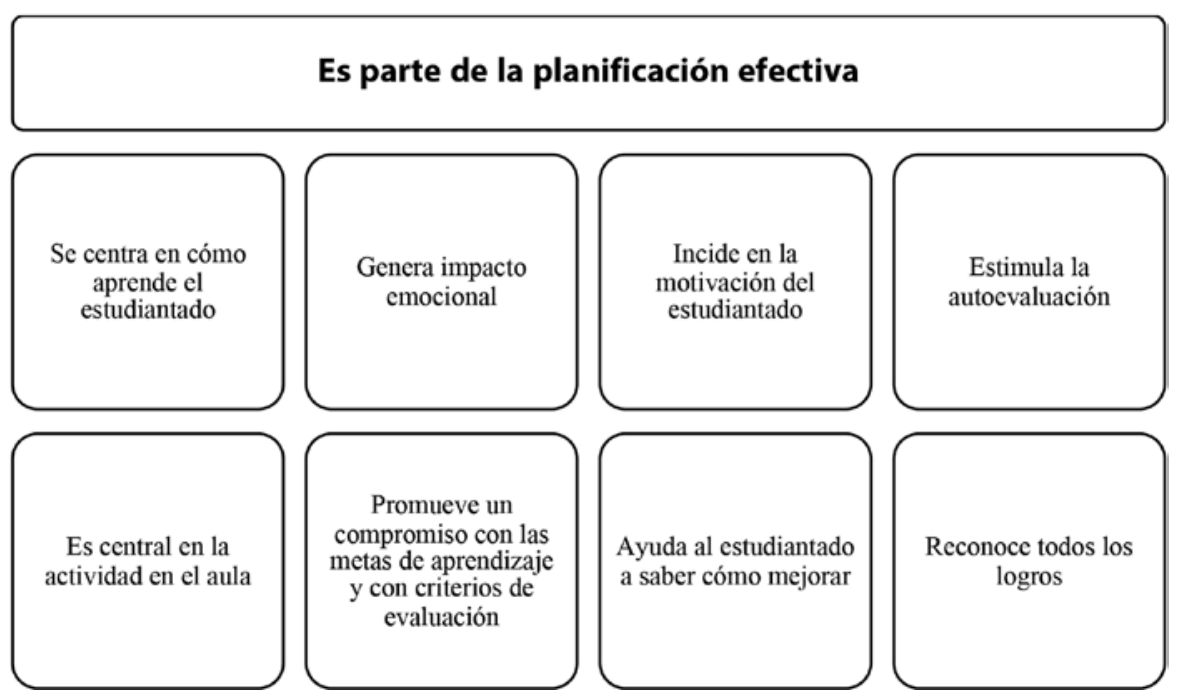

Figura 2: Ideas en la evaluación de los aprendizajes en contexto multiculturales (Flores, 2017)

Es parte de la planificación efectiva, se debe tener criterios de evaluación claramente definidos, a partir de los cuales se planifique lo que se realizará en cada clase y se monitoreen permanentemente los logros de los estudiantes. Se centra en cómo aprende el estudiantado, es importante que el profesorado no solamente se centre en qué aprende el estudiantado, sino también en cómo lo aprenden, teniendo en cuenta que ellos sean cada vez conscientes de sus propios procesos de aprendizaje. Es central en la actividad de aula, la evaluación es algo que se planifica en base a aprendizaje cuyo logro se monitorea permanentemente y no un accesorio que aparece solamente al final de los procesos de aprendizaje. Genera impacto emocional, es importante cuidar los comentarios que se hace al estudiantado, porque ello incide en su autoestima.

Incide en la motivación del estudiantado, en directa relación con lo anterior, es necesario considerar que un estudiante puede llegar a rechazar un subsector solamente por las calificaciones que obtiene en él, pues llega a convencerse de que "es malo en tal tarea”. De ahí la importancia de entregar una buena retroalimentación (feedbacks), que ayude al estudiante a entender sus logros, sus dificultades y las formas de mejorar su aprendizaje. Solamente de esta forma entenderá que su mala calificación "no es perpetua", sino que existe la posibilidad de regresar si se mejora lo que el profesor sugiere. Promueve un compromiso con las metas de aprendizaje y con criterios de evaluación, al existir criterios bien definidos y compartidos con el estudiantado, ellos pueden llegar a comprometerse con lo que hay que lograr, pues sienten que lo que hacen tiene un sentido. Cuando el estudiantado pregunta "¿y para qué estamos haciendo esto?", significa que las metas no están claras y hay que hacer algo al respecto.

Ayuda al estudiantado a saber cómo mejorar, una buena retroalimentación (feedbacks) no solamente indica al estudiante en qué se equivocó, sino también en qué 
estuvo bien y, sobre todo, cómo puede mejorar aquello en que presentó debilidades. Estimula la autoevaluación, al promoverse el compromiso con metas de aprendizaje por parte de los estudiantes, resulta natural que se los haga transitar paulatinamente desde la heteroevaluación a la autoevaluación, pues a través de ello se promueve que sean capaces de monitorear sus propios procesos. Reconoce todos los logros, muchas veces se tiene tan presente el nivel de desempeño más alto (el estudiantado sobresaliente), que se olvida reconocer los avances intermedios, haciendo sentir a los demás estudiantes que su desempeño fue 'malo'. Por eso, es importante reconocer la variedad de aprendizajes en el aula, valorar los diferentes niveles de logro alcanzados y apoyar todos los avances observados, incluso los más pequeños.

Por tanto, el propósito de la educación es responder a las necesidades de potenciar el desarrollo integral de cada estudiante y a la necesidad de producir y reproducir la parte cultural de la comunidad, se debe plantear en la universidad el enfoque cualitativo de la evaluación donde el evaluador debe tratar de interpretar el significado de las conductas y no simplemente establecer cantidad y tipo de conducta (Arévalo, Pardo, \& Vigil, 2016).

En la evaluación del aprendizaje desde una perspectiva intercultural, se pretende introducir estrategias y procedimientos evaluativos diferentes a las que han predominado en la educación formal. Se trata de un intento de mejorar las posibilidades de aprender utilizando la evaluación como un medio y ayuda, así como rescatando las formas de valoración que tienen las comunidades indígenas, afrodescendientes, mestiza y otras comunidades étnicas. En tal sentido, la evaluación debe ser un medio que permita asegurar que las estrategias elegidas para evaluar sean adecuadas y considere los propósitos formativos, respetando las características propias del estudiantado, en definitiva, que sea una respuesta a un contexto sociocultural determinado, que asegure el éxito en el aprender.

La evaluación de los aprendizajes desde una perspectiva intercultural se centra fundamentalmente en recoger vivencias y evidencias sobre el aprendizaje de procesos más que de resultados, y se interesa en que sea el propio estudiante quien asuma responsabilidades. También, la evaluación intercultural debe ser un diálogo de culturas, donde se incorpore criterios de valoración de los aprendizajes de las comunidades indígenas, afrodescendientes y mestizo, dicha de otra forma la valoración de las personas en su desarrollo comunitario. Por ejemplo, las comunidades étnicas de Nicaragua, inician un proceso investigación y validación para incorporar un nuevo saber en su cotidiano vivir. Este proceso de investigación y validación es controlado por la propia comunidad en las distintas labores que realizan los miembros de esta comunidad. Es decir, cuando se trata de valorar los conocimientos, saberes y prácticas la comunidad establece parámetros, donde la observación y el diálogo armónico ayuda a desarrollar potencialidades que necesitan la juventud para desenvolverse en una sociedad. 


\subsection{La evaluación de los aprendizajes desde una perspectiva intercultural de género}

La evaluación de los aprendizajes desde una perspectiva intercultural de género se cimienta en principios claves de evaluación de las prácticas en el aula intercultural, que puntualizan aspectos como:

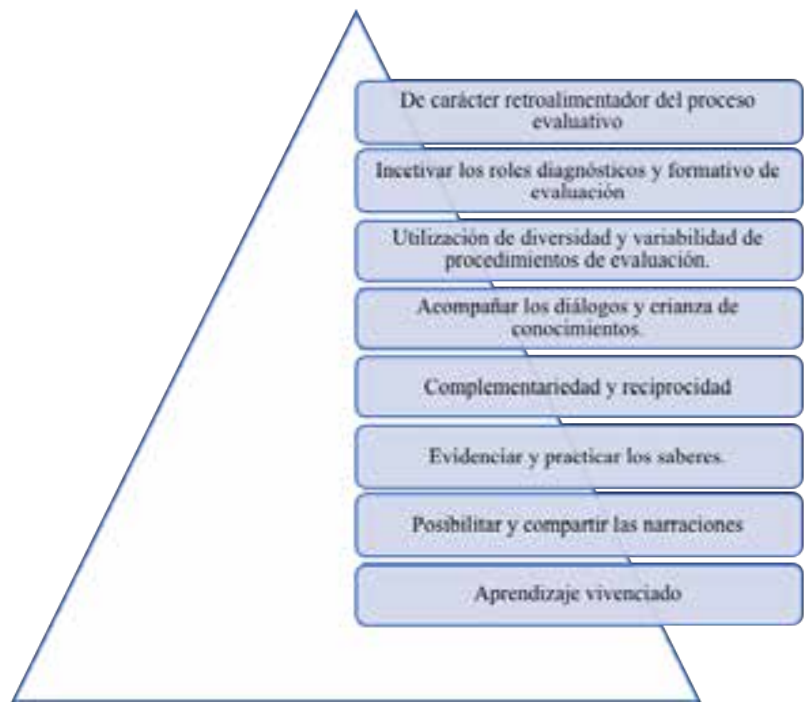

Figura 3: Principios de evaluación de las prácticas en el aula intercultural (Flores, 2017).

De carácter retroalimentador del proceso evaluativo. El profesor favorece la activación de conocimientos previos del estudiantado, cuando verifica que sus esquemas cognitivos establecen conexiones con los nuevos conocimientos, cuando apoya el proceso cognitivo a través de observar sus logros y retroalimentarlos, hacer regulaciones pedagógicas para fomentar nuevos avances, facilitar la autoevaluación del estudiantado y su toma de conciencia respecto a lo aprendido y a la falta por aprender. Esto obliga al profesorado a estar siempre atento a las posibles carencias o desviaciones que sufren los diferentes procesos de formación. Por otra parte, también implica aceptar la presencia del error como una forma natural en la concreción de nuevo aprendizaje y que no necesariamente conduzca a una determinada sanción.

De incentivar los roles diagnósticos y formativos de evaluación. El proceso evaluativo es una concepción centrado en el alcance de los aprendizajes donde se enfatiza los roles de diagnóstico y formativo, entendiéndolo como logros o resultados en el sentido del estudiantado. Además, como un proceso legitimado curricularmente y didácticamente, garantizado los roles de diagnóstico, formativo y sumativa.

De la utilización de la diversidad y variabilidad de procedimientos de evaluación. La utilización de nuevos procedimientos evaluativos complementa la información que se quiere vivenciar del desempeño del estudiantado. Aceptar, por ejemplo, que la 
utilización de técnicas e instrumentos que aportan información sobre el aprendizaje de los estudiantes debería ser considerado como un procedimiento de evaluación, como los mapas semánticos, los mapas conceptuales, los gráficos síntesis, listas de cotejo, guías de observación, indicadores de logros o desempeño, etc. Además, la utilización de estos implica la flexibilidad en su construcción, cuando las circunstancias nos dicen hay que variar el proceso, dando una apertura a la diversidad y variabilidad que son atributos inseparables de las comunidades indígenas, afrodescendientes y mestizas.

De carácter de acompañar los diálogos y crianza de conocimientos, saberes y prácticas. El acompañamiento y crianza de conocimientos significa compartir saberes, narrar las propias vivencias y aprender de las experiencias de las comunidades étnicas. Por lo tanto, se trata de una actitud de escucha, de observación y de dialogar en lo que se está dispuesto a reconocer o encontrarse con el otro, sin afanes de imponer la verdad, sino con la disposición de dialogar para confluir en el mismo ritmo de fluir de la vida. El acompañamiento obliga a estar en la cotidianidad de la comunidad incorporando sus conocimientos, saberes y prácticas, donde le profesorado son sujetos que establecen diálogos armónicos en complementariedad con los demás.

De carácter complementariedad y reciprocidad. Entendida la complementariedad como la comprensión y actuación de forma conjunta y colaborativa que conlleva a la unidad y equilibro entre los individuos y su entorno para potenciar las capacidades y alcanzar los logros de aprendizaje. En este sentido, se identifican las singularidades de aprendizaje del estudiantado que se complementan para versar el mismo ritmo, es una confluencia entre diversos estudiantes, más que una homogenización de acuerdos. Esto significa una apertura a los eventos e intervenciones educativas más desequilibrio cotidiano.

De posibilitar y compartir las narraciones. Se trata de evidenciar los conocimientos, saberes y prácticas de cada individuo con relación a su colectividad. Es importante trabajar con narraciones en los coloquios porque ayuda al estudiantado a explicar sus aprendizajes con sus experiencias vividas en la comunidad.

Partir de un aprendizaje vivenciado en comunidad. El estudiantado aprende a través de las experiencias en comunidad. Esta experiencia permite explicar los procesos de intersubjetividad en los aprendizajes y creación y re-recreación de los conocimientos, saberes y prácticas. Las experiencias son acciones de aprendizaje cotidiano que se dan, ya sea con la intervención en la comunidad o fuera de ella.

\section{Conclusiones y Perspectivas futuras}

La educación intercultural se caracteriza por favorecer dinámicas inclusivas en todos los procesos de socialización, aprendizaje y convivencia dentro del ámbito educativo. Además, ayuda a desarrollar competencias y actitudes para la participación activa en 
la construcción de una sociedad plurilingüe, pluricultural, justa y equitativa. Desde esta perspectiva, la enseñanza y aprendizaje desde el enfoque intercultural, es una estrategia para promover espacios de innovación de nuevos saberes y haceres, permitiendo la confrontación de conocimientos desde diferentes horizontes y perspectivas culturales, abriendo la posibilidad de impulsar procesos de complementariedad y enriquecimiento entre la ciencia y los saberes de los pueblos. Entonces, en la evaluación de los aprendizajes, se tiene punto de partida la comunidad y los saberes de pueblos, estos son:

- Saber Ser. Es la capacidad de construir una comunidad de aprendizaje, teniendo de referencia la interculturalidad; la perspectiva intercultural de género; la autonomía regional; buen vivir y desarrollo con identidad; y el dialogo de saberes y haceres con la finalidad de crear y recrear conocimientos y experiencias de los pueblos.

- Saber Hacer. Es aprender en comunidad, se trata de promover el aprendizaje dialógico; un aprendizaje cooperativo; un aprendizaje socioafectivo; un aprendizaje basado en problema; y un aprendizaje autónomo con el propósito de innovar y emprender en y con la comunidad para generar ambientes de aprendizajes interculturales y pluritecnológicos y sistemas de prácticas compartidas.

- Saber Conocer. Es formar en y para la comunidad (perfil de formación), se parte del modelo pedagógico de universidad comunitaria intercultural retomando los conocimientos, habilidades y actitudes en las acciones formativas; y las estrategias de seguimiento de evaluación en los aprendizajes impulsando el papel que juega la comunidad en la universidad y la búsqueda de nuevos paradigmas en los enfoques pedagógicos y metodológicos para la generación de conocimientos para el buen vivir de los pueblos.

- Saber convivir. Es acompañar a la comunidad con objetivos y contenidos relevantes, significantes y propios de los pueblos. Se incorporan los conocimientos, habilidades y actitudes en metodologías activas y participativas con una evaluación que retroalimenta y se mejora con la reflexión, todo ello, con el propósito de alcanzar resultados de aprendizaje que evidencia actividades que proporcionan conocimientos, comprensión, aplicación, análisis, síntesis y evaluación de los saberes y haceres de los pueblos.

En este sentido, la evaluación de los aprendizajes se presenta como una posibilidad de desplazamiento de perspectiva en contra de las prácticas más habituales en evaluación. La evaluación de los aprendizajes es parte de la planificación efectiva; se centra en como aprende el estudiantado; generar impacto emocional; incide en la motivación del estudiantado; estimula la autoevaluación; es central en la actividad en el aula; promueve un compromiso con las metas de aprendizaje y con criterios de evaluación; ayuda al estudiantado a saber cómo mejorar; y reconoce todos lo logros. 
Además, la evaluación de los aprendizajes en la educación intercultural es de carácter retroalimentador del proceso evaluativo; de incentivar los roles diagnósticos y formativo de evaluación; de la utilización de la diversidad y variabilidad de procedimientos de evaluación; de carácter de acompañar los diálogos y crianza de conocimientos, saberes y prácticas; de carácter complementariedad y reciprocidad; de posibilitar y compartir las narraciones; $y$ tiene que partir de un aprendizaje vivenciado en comunidad.

Se concluye, que una evaluación de los aprendizajes inclusiva para poblaciones en contextos multiculturales, tiene que contribuir a la reducción de situaciones de desigualdad y exclusión social, así como, promover una evaluación para todos y con todos, teniendo de referencia el reconocimiento de la diversidad de saberes y prácticas, y la incorporación de tecnologías accesibles que posibiliten un aprendizaje para toda la vida.

\section{Lista de referencias}

Amador-Núñez, F., Reyes-Gómez, M., \& Flores, W. O. (2015). Metodologías en la enseñanza del cálculo de probabilidades en undécimo grado de educación secundaria. Ciencia e Interculturalidad, 17(2), 15-27. DOI: https://doi.org/10.5377/ rci.v17i2.2637

Arévalo, I., Pardo, K., \& Vigil, N. (2016). Enseñanza de castellano como segunda lengua en las escuelas EIB. Recupero el 15 de mayo de 2018 en: www.aulaintercultural.org

Banco Mundial. (2018). Aprender para hacer realidad la promesa de la educación. Washington: World Bank. https://openknowledge.worldbank.org/handle/10986/28340

Flores, W. (2015). Los problemas asociados a la comprensión del álgebra en estudiantes universitarios. Horizontes pedagógicos, 17(1), 8-23.

Flores, W. O., \& Gutiérrez y Restrepo, E., León, O., Sarraipa, J., Pantoja, C., Merino, C., Calderón, D., Guinocchio, M., Rivera, M., Calderón, M., E., \& Boticario, J., G. (2016). Centros de Apoyo y Desarrollo Educativo Profesional para la observación y disminución de la deserción universitaria. Ciencia e Interculturalidad, 18(1), 48-62. DOI: http://dx.doi.org/10.5377/rci.v18i1.3049

Flores, W. O., \& Rojas, N. (2013). Integración de las tecnologías de la información y comunicación en la enseñanza universitaria. Horizontes pedagógicos, 15(1), 46-57.

Flores, W. O., \& Salinas, M. J. (2012). Metodologías utilizadas en la enseñanza-aprendizaje de la derivada: influencia en el rendimiento de la asignatura "Matemática Financiera”. Horizontes pedagógicos, 14(1), 9-20. DOI: https://doi.org/10.5377/ rci.v12i1.1215

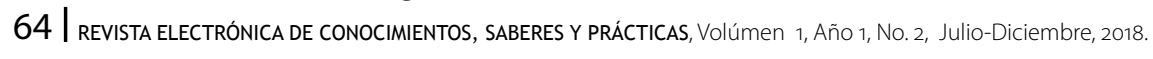


Flores, W., \& Auzmendi, E. (2015). Resolución de problemas matemáticos: un cuestionarios para su evaluación y comprensión. Ciencia e Interculturalidad, 16(1), 54-74. DOI: https://doi.org/10.5377/rci.v16i1.2353

Flores, W., O. (2017). Sistema de evaluación de los aprendizajes desde la pedagogía intercultural. Managua: URACCAN.

Flores, W., O., \& Olivar-Molina, S. (2016). Actitudes hacia la estadística en la formación del profesorado para contextos multiculturales. Revista Universitaria, 17(2), 27-37. DOI: http://dx.doi.org/10.5377/ruc.v17i2.3235

Gaitán-Hernández, M., Lacayo-Robles, M., \& Flores, W. O. (2014). La parábola en undécimo grado aplicando el modelo de Van Hiele. Ciencia e Interculturalidad, 15(2), 21-33. DOI: https://doi.org/10.5377/rci.v15i2.1917

López-Mairena, E., \& Trillo-Alonso, J. (2013). Evaluación de las competencias en los estudiantes, URACCAN-Nueva Guinea. Ciencia e Interculturalidad, 12(1), 22-38. DOI: https://doi.org/10.5377/rci.v12i1.1214

Roncal, F. (2005). Evaluación de los aprendizajes. Guatemala: La Salle.

Santos-Guerra, M. (1999). La evaluación: Un proceso de diálogo comprensión y mejora. Río de la Plata: La Salle.

Tobón, S. (2008). La formación basada en competencias en la educación superior: el enfoque complejo. México: Universidad Autónoma de Guadalajara. 